\title{
Determination of Intrinsic Dissolution Rate Using Miniaturized Rotating and Stationary Disk Systems
}

\author{
Yin-Chao Tseng ${ }^{1, *}$, Mita Patel ${ }^{1}$, and Youna Zhao ${ }^{2}$ \\ 'Boehringer Ingelheim Pharmaceuticals, Inc., 900 Ridgebury Road, Ridgefield, CT 06877 \\ ${ }^{2}$ Bright Future Pharmaceutical Laboratories Ltd., 8 Wang Fu Street, Yuen Long, N.T., Hong Kong
}

\begin{abstract}
The objective of this study was to assess the feasibility and reliability of using miniaturized disk dissolution apparatus for intrinsic dissolution rate (IDR) measurement in the early drug discovery process when bulk drug supply is very limited. The two apparatus, a miniaturized rotating disk system (mRDS) and a miniaturized stationary disk system (mSDS), were evaluated using chloramphenicol as a model drug with sample sizes of 3-10 mg. Wood's rotating disk system (wRDS) was used as control with a sample size of $150 \mathrm{mg}$.

The effect of various experimental parameters on IDR was studied on the two miniaturized apparatus. While compression force, disk distance, dissolution volume, and drug loading did not have significant effects on IDR measured by mRDS, dissolution volume and disk distance showed significant effects by mSDS. When all experimental parameters were held constant, the stationary system generated significantly higher IDRs compared with the two rotating systems (mRDS and wRDS). The mRDS yielded IDR values comparable to those by wRDS at $25 \mathrm{rpm}$ (a slower rotation speed).

These study results indicate that both miniaturized systems produce reliable IDR measurements with a small quantity of material, which provides a desirable advantage over other methods (e.g., wRDS, solubility measurement) in the early drug discovery phase.
\end{abstract}

KEYWORDS: Intrinsic dissolution; dissolution apparatus; rotating disk dissolution; stationary disk dissolution.

\section{INTRODUCTION}

$\mathrm{O}$ ne of the key phases in drug discovery research is the period from lead optimization to solid form selection for a new active pharmaceutical ingredient (API). During this phase, the physicochemical properties of drug substances are evaluated and optimized, and the lead solid form of a development candidate is selected. Of the physicochemical properties, the solubility and dissolution rate are particularly important for lead optimization, since they are critical parameters affecting drug absorption (1). The dose number $\left(D_{0}=\right.$ dose $/ 250 \mathrm{~mL} /$ solubility $)$, which takes projected human dose into consideration in the Biopharmaceutics Classification System (BCS), is frequently used for classifying a drug substance based on solubility $(2,3)$. For the determination of dissolution rate, the intrinsic dissolution rate (IDR) measurement, where the dissolution surface area is kept constant, is the method of choice. This is because the particle size, and consequently the surface area, of a drug substance are not well controlled at the drug discovery stage. Determination of the IDR of a compound very early in the process enables successful screening of drug candidates and their solid forms, and it also helps with understanding of dissolution behavior of a compound under different biophysiological $\mathrm{pH}$ conditions $(4,5)$.

${ }^{*}$ Corresponding author.
IDR is defined as the dissolution rate of a pure compound under the condition of constant surface area. IDR is determined according to the equation (6)

$$
\mathrm{IDR}=(d m / d t)_{\max } / A
$$

where the units of IDR are $\mathrm{mg} / \mathrm{min} / \mathrm{cm}^{2}, A$ is the area of the drug disk $\left(\mathrm{cm}^{2}\right), m$ is the mass $(\mathrm{mg}), t$ is the time (min), and $(d m / d t)_{\max }$ is the maximum slope in the dissolution curve evaluated at the start of the dissolution process. IDR is affected by the solid-state properties of a drug substance and is also influenced by external factors, such as compression force and hydrodynamics (e.g., dissolution vessel, dissolution medium volume, die position, and rotation speed). The intrinsic dissolution apparatus provides a convenient way to measure accurate IDR by exposing a drug substance under study to a constant surface of dissolution medium. There are two types of intrinsic dissolution systems, the stationary disk system (SDS) and the rotating disk system (RDS) $(7,8)$. SDS is described in the USP, and RDS in the USP, EP, and BP (9-11). The Wood's rotating disk system (wRDS) is a standard apparatus used for measuring IDR (12), where approximately $150-500 \mathrm{mg}$ of a drug substance is compressed inside a die with a punch to produce a drug disk with an exposed surface area of $0.5 \mathrm{~cm}^{2}$. During dissolution, the exposed area of the drug disk is kept constant, and the temperature of the dissolution baths is kept at $37^{\circ} \mathrm{C}$, accommodating volume of $900 \mathrm{~mL}$ in each 
vessel. Standard dissolution apparatus require a large quantity of drug substance, which is not usually available in the drug discovery stage.

An ideal dissolution apparatus for an early discovery phase would require a much smaller sample size $(<10 \mathrm{mg})$, yet enable IDR measurements as reliable as the standard dissolution apparatus. The objective of this study was to determine if a miniaturized rotating disk system (mRDS), a miniaturized stationary disk system (mSDS), or both can be used as an intrinsic dissolution system ideal for the discovery setting. To compare the performance of three different dissolution systems (WRSD, mRSD, and mSDS), a model drug, chloramphenicol, was selected for the studies on the basis of its adequate solubility $(1.5 \mathrm{mg} / \mathrm{mL})$ predetermined in $0.1 \mathrm{M}$ citrate-phosphate buffer ( $\mathrm{pH} 4.5)$ at $37^{\circ} \mathrm{C}$. The effects of various parameters (compression force, dissolution medium volume, drug loading, die distance, and rotation speed) on IDR were also studied.

\section{EXPERIMENTAL \\ Materials}

Chloramphenicol was purchased from Sigma-Aldrich (St. Louis, MO, USA). All other chemicals were analytical reagent grade or equivalent and purchased commercially. The $0.1 \mathrm{M}$ citrate-phosphate buffer ( $\mathrm{pH} 4.5)$, in which chloramphenicol showed an adequate solubility suitable for the studies, was prepared according to the European Pharmacopeia procedure with the modification of mixing $6.3 \mathrm{~L}$ of $0.1 \mathrm{M}$ anhydrous disodium hydrogen phosphate and $4 \mathrm{~L}$ of $0.1 \mathrm{M}$ citric acid (13).

\section{Apparatus}

All dissolution studies were performed with a Distek dissolution unit 2100C and an Evolution 4300 autosampler (Distek Inc., NJ, USA). Dissolution vessels, rotating and stationary disk systems were either purchased from or custom made by Distek, Inc., or fabricated by the in-house machine shop at Boehringer Ingelheim Pharmaceuticals,
Inc. The mini apparatus were based on the USP setup but scaled down with respect to the dimensions. The die cavity diameter, drug loading, dissolution vessel size, and dissolution medium volume of the miniaturized systems were scaled down as summarized in Table 1. Figure 1 shows examples of wRDS, mRDS, and mSDS apparatus. A mini-compression system (Distek Inc., NJ, USA) was used to make drug disks. As little as 3-10 mg of chloramphenicol powder was loaded into the cylindrical hole of a passivated stainless steel die and compressed (90 sec at 100-300 psi) to a flat surface with an exposed area of $0.0314 \mathrm{~cm}^{2}$ (Figure 2).

\section{Intrinsic Dissolution Testing}

The standard intrinsic disk dissolution parameters were selected based on the USP testing conditions (9). The following parameters for the miniaturized systems were studied as shown in Table 1: compression force (100-300 psi), die distance (0.5-1.5 in), drug loading (3-10 mg), and rotation speed (50-125 rpm). The dissolution medium volume was $50-200 \mathrm{~mL}$ in a 100 - or 200-mL vessel. Experiments were run at $37^{\circ} \mathrm{C}$, and $0.1 \mathrm{M}$ citrate-phosphate buffer ( $\mathrm{pH} 4.5$ ) was used as the dis-

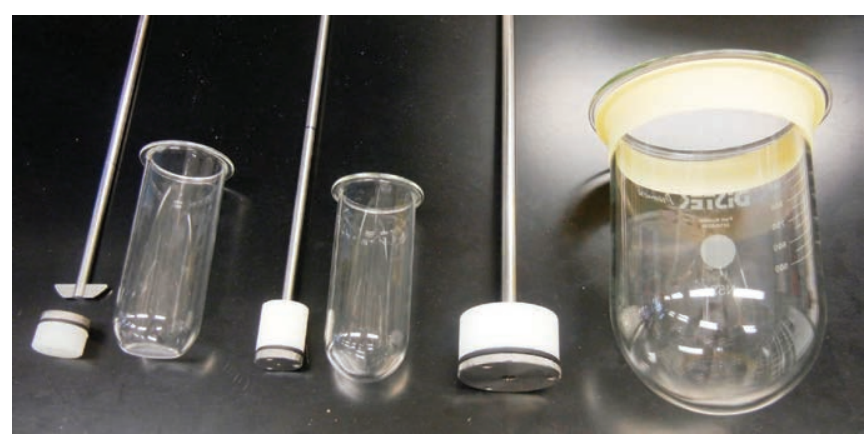

Figure 1. Miniaturized stationary disk system, miniaturized rotating disk system, and Wood's rotating disk system (left to right).

Table 1. Experimental Conditions and Parameters for Intrinsic Dissolution Studies on Chloramphenicol

\begin{tabular}{|c|c|c|c|}
\hline Parameter & $\begin{array}{l}\text { Wood's rotating disk system } \\
\text { (wRDS) }\end{array}$ & $\begin{array}{l}\text { Miniaturized rotating disk system } \\
\text { (mRDS) }\end{array}$ & $\begin{array}{l}\text { Miniaturized stationary disk system } \\
\text { (mSDS) }\end{array}$ \\
\hline Vessel size & 1000-mL round bottom & $100-\mathrm{mL}$ round bottom & 100-200-mL flat bottom \\
\hline Dissolution Medium volume $(\mathrm{mL})$ & 900 & $50-100$ & $50-200$ \\
\hline Medium & $\begin{array}{c}0.1 \mathrm{M} \text { citrate-phosphate buffer; } \\
\mathrm{pH} 4.5\end{array}$ & $\begin{array}{l}0.1 \text { M citrate-phosphate buffer; } \\
\text { pH } 4.5\end{array}$ & $\begin{array}{l}0.1 \text { M citrate-phosphate buffer; } \\
\text { pH } 4.5\end{array}$ \\
\hline Die cavity diameter/area & $0.8 \mathrm{~cm} / 0.5024 \mathrm{~cm}^{2}$ & $0.2 \mathrm{~cm} / 0.0314 \mathrm{~cm}^{2}$ & $0.2 \mathrm{~cm} / 0.0314 \mathrm{~cm}^{2}$ \\
\hline Drug loading & $150 \mathrm{mg}$ & $3-10 \mathrm{mg}$ & $5 \mathrm{mg}$ \\
\hline Compression force/ holding time & $300 \mathrm{psi} / 90 \mathrm{sec}$ & $100-300 \mathrm{psi} / 90 \mathrm{sec}$ & $100-300 \mathrm{psi} / 90 \mathrm{sec}$ \\
\hline Disk distance (in) & 1 & $0.5-1.5$ & $0.5-1.5$ \\
\hline Rotation speed (rpm) & $50-125$ & $50-100$ & $50-100$ \\
\hline Temperature $\left({ }^{\circ} \mathrm{C}\right)$ & 37 & 37 & 37 \\
\hline Detection & HPLC & HPLC & HPLC \\
\hline
\end{tabular}


solution medium. Chloramphenicol was used as a model compound. An aliquot of the solution was withdrawn at $5,10,15,20,30,45$, and 60 min and filtered using a 0.45- $\mu \mathrm{m}$ PVDF syringe filter (Whatman, New Jersey, USA). The filtered samples were analyzed by an Agilent 1100 series HPLC system (Santa Clara, USA) equipped with a $150 \times 4.6 \mathrm{~mm}$ Agilent Zorbax Rx-C18 column. The mobile phases consisted of acetonitrile and water with $0.1 \%$ trifluoroacetic acid. A gradient elution method with a flow rate of $0.6 \mathrm{~mL} / \mathrm{min}$ was employed with detection at $234 \mathrm{~nm}$ via a photodiode array detector. The injection volume was $25 \mu \mathrm{L}$. The retention time of chloramphenicol was approximately $4.9 \mathrm{~min}$. The mean and standard deviations were calculated at each time point for $n=6$ vessels. The IDR of chloramphenicol in $\mathrm{mg} / \mathrm{min} / \mathrm{cm}^{2}$ was determined from the slope of the regression line on first four time points $(5,10,15$, and $20 \mathrm{~min})$. The percentage difference in IDR and statistical significance of difference by $t$-test were used to compare the intrinsic dissolution profiles generated by the systems.

\section{RESULTS AND DISCUSSION}

The drug release rate of chloramphenicol was investigated at various conditions using different intrinsic dissolution systems (wRDS, mRDS, and mSDS). When plotted against time $(5,10,15$, and 20 min time points), the amount of drug dissolved per unit area produced a linear curve with correlation coefficients greater than 0.996 in each case. The \%CV is less than $10 \%$, indicating good reproducibility. The representative IDR values of chloramphenicol measured using three dissolution systems at $100 \mathrm{rpm}$ rotation speed at $\mathrm{pH} 4.5$ are illustrated in Figure 3. The IDR values of chloramphenicol are 0.403 , 0.458 , and 0.944 measured by wRDS, mRDS, and mSDS, respectively.

When compared with the IDR of wRDS as the control, the IDR value increased by $12.8 \%$ with $\mathrm{mRDS}$ and by $80.3 \%$ with mSDS. The difference in the IDR values between rotating (mRDS and wRDS) and stationary (mSDS) systems is substantial, which may be attributed to the difference in hydrodynamics and dissolution mechanisms (14-16). The dissolution of stationary disk systems (similar to USP Apparatus 2) is more dominant with the convection dissolution mechanism, and that of rotating disk systems (similar to USP Apparatus 1) is achieved by shear-like motion. The convection dissolution mechanism increases the hydrodynamics of dissolution medium flow and reduces the diffusion layer, which increases the dissolution of disk compounds (17). On the other hand, compared with mSDS, the difference in IDR between mRDS and wRDS is relatively less noticeable, suggesting that the hydrodynamics and dissolution mechanism in the miniaturized rotating disk system are similar to that of the standard Wood's disk system.

The effect of various parameters on IDR of chloramphenicol was further investigated. Tables 2-4 compare

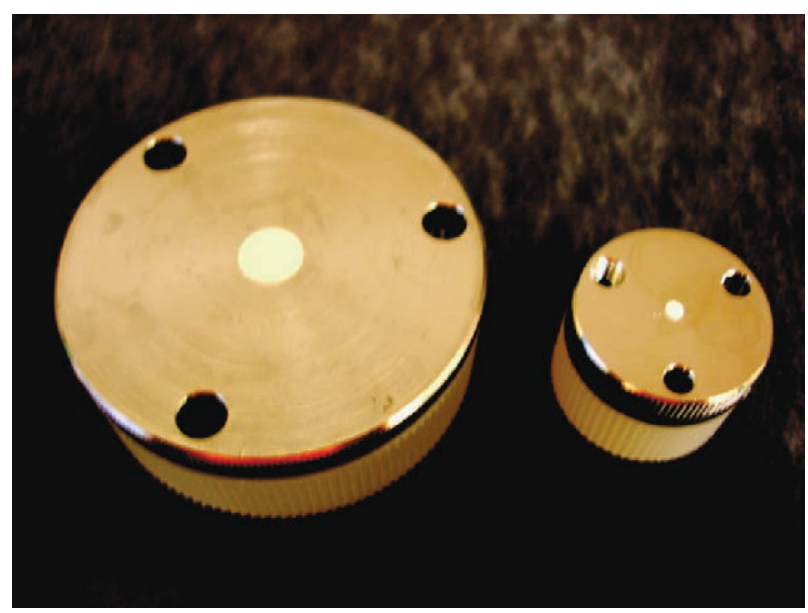

Figure 2. Standard and miniaturized dies.

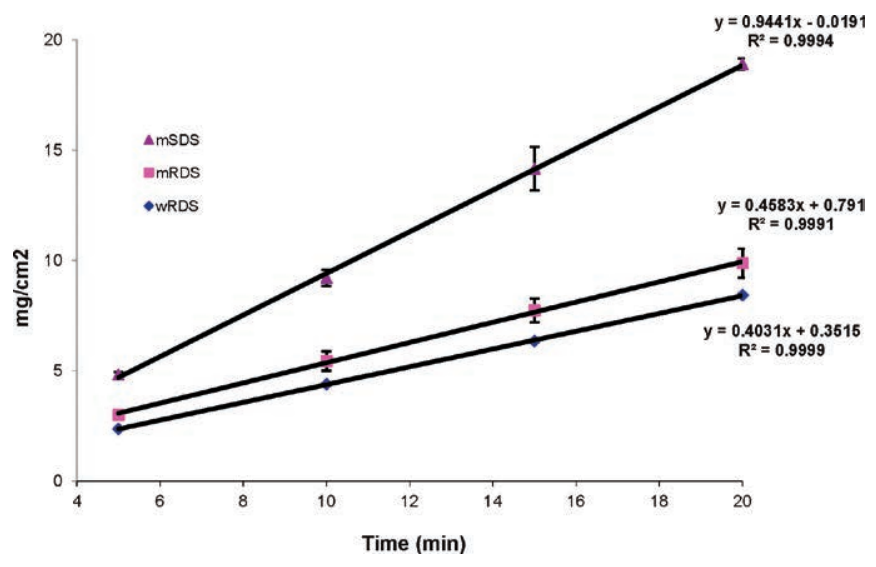

Figure 3. Intrinsic dissolution curves of chloramphenicol measured by $w R D S, m R D S$, and mSDS at 100-rpm rotation speed. Compression force: 300 psi, distance: 1 in, volume/vessel: 50/100 mL, drug loading: 5 mg, $n=6$.

the IDR of chloramphenicol tested employing different compression forces, disk distances, dissolution medium volumes, amounts of drug loading, and rotation speeds using wRDS, mRDS, and mSDS. The differences in IDR values were compared by percentage difference as well as by $t$-test.

\section{Disk Compression Force}

The effect of disk compression force on IDR of chloramphenicol was investigated, and the disk compression forces ranged from 100 to 300 psi for both mRDS and mSDS systems. Other parameters were kept constant: disk at 1-in distance, $50 \mathrm{~mL}$ dissolution medium in a $100-\mathrm{mL}$ vessel, 5-mg drug loading, and 50-rpm rotation speed for both systems. The 300-psi compression force was used as the control for the comparison of differences in IDR. As shown in Tables 2 and 3, when powder was compressed into drug disks with different compression forces, there 
was no statistical difference in IDR for both sets of experiments (i.e., the mRDS set and the mSDS set).

\section{Disk Distance}

The effect of disk distance (from the disk surface to the bottom of the vessel for mRDS or from the disk surface to the paddle blade for mSDS) on the IDR of chloramphenicol was also investigated. The distances studied included 0.5, 1 , and 1.5 in for mRDS and 1 and 1.5 in for mSDS. Other parameters were kept constant: 300-psi compression force, $50-\mathrm{mL}$ dissolution volume in a $100-\mathrm{mL}$ vessel, 5 -mg drug loading, and 50-rpm rotation speed for both systems. The 1-in distance was used as control for the comparison of differences in IDR. As shown in Table 2, when mRDS was used, the percentage difference in IDR was less than $10 \%$ compared with the control, and there was no statistical difference. However, as shown in Table 3, when mSDS was used, the disk distance had a significant effect on IDR.

\section{Dissolution Medium Volume}

The effect of dissolution medium volume $(50,100$, or $200 \mathrm{~mL}$ ) in a 100- or 200-mL vessel on IDR of chloramphenicol was studied for both mRDS and mSDS systems. Other parameters were kept constant: 1-in disk distance, 300-psi compression force, 5-mg drug loading, and 50rpm rotation speed for both systems. As shown in Table 2, when a 50- or 100-mL dissolution medium volume in a $100-\mathrm{mL}$ vessel and $\mathrm{mRDS}$ were used, the percentage difference between the two volumes in IDR was $7 \%$ (no statistically significant difference). However, as shown in Table 3, when mSDS was used, the volume of medium had a significant effect on IDR. The increase in medium volume from 50 to $100 \mathrm{~mL}$ in a $100-\mathrm{mL}$ vessel or to $200 \mathrm{~mL}$ in a $200-\mathrm{mL}$ vessel resulted in a much greater percentage difference (13-25\%) with a statistically significant difference in IDR. This suggests that for mSDS, the hydrodynamic changes are more affected by

Table 2. Effect of Compression Force, Drug Loading, Disk Distance, and Dissolution Volume on IDR of Chloramphenicol Measured by $m R D S^{a}$

\begin{tabular}{|c|c|c|c|c|c|c|c|c|}
\hline $\begin{array}{l}\text { Compression force } \\
\text { (psi) }\end{array}$ & $\begin{array}{l}\text { Drug loading } \\
\text { (mg) }\end{array}$ & $\begin{array}{l}\text { Distance } \\
\text { (in) }\end{array}$ & $\begin{array}{l}\text { Media volume/ } \\
\text { Vessel size (mL) }\end{array}$ & $\begin{array}{c}\text { IDR } \pm \text { SD } \\
\left(\mathrm{mg} / \mathrm{cm}^{2} / \mathrm{min}\right)\end{array}$ & $\begin{array}{l}\text { CV } \\
(\%)\end{array}$ & $R^{2}$ & $\begin{array}{l}\text { IDR percentage } \\
\text { difference (\%)* }\end{array}$ & $t$-test $^{b}$ \\
\hline 300 & 5 & 1.0 & $50 / 100$ & $0.362 \pm 0.023$ & 6.33 & 0.9993 & Control & Control \\
\hline 250 & 5 & 1.0 & $50 / 100$ & $0.335 \pm 0.022$ & 6.57 & 0.9997 & 7.8 & Not significant \\
\hline 200 & 5 & 1.0 & $50 / 100$ & $0.371 \pm 0.010$ & 2.71 & 0.9983 & 2.4 & Not significant \\
\hline \multirow{6}{*}{100} & 5 & 1.0 & $50 / 100$ & $0.351 \pm 0.027$ & 7.72 & 0.9974 & 3.2 & Not significant \\
\hline & 5 & 0.5 & $50 / 100$ & $0.388 \pm 0.015$ & 3.99 & 0.9987 & 7.0 & Not significant \\
\hline & 5 & 1.5 & $50 / 100$ & $0.381 \pm 0.025$ & 6.45 & 0.9993 & 5.0 & Not significant \\
\hline & 5 & 1.5 & $100 / 100$ & $0.337 \pm 0.021$ & 6.12 & 0.9975 & 7.1 & Not significant \\
\hline & 3 & 1.5 & $100 / 100$ & $0.345 \pm 0.016$ & 4.77 & 0.9994 & 4.7 & Not significant \\
\hline & 10 & 1.5 & $100 / 100$ & $0.380 \pm 0.022$ & 5.90 & 0.9986 & 4.9 & Not significant \\
\hline
\end{tabular}

Rotation speed: 50 rpm; $n=6$.

${ }^{b} p<0.05$; IDR of sample is significantly different from that of control.

Table 3. Effect of Compression Force, Disk Distance, and Dissolution Volume on IDR of Chloramphenicol Measured by mSDSa

\begin{tabular}{|c|c|c|c|c|c|c|c|c|}
\hline $\begin{array}{l}\text { Compression force } \\
\text { (psi) }\end{array}$ & $\begin{array}{l}\text { Drug Loading } \\
\text { (mg) }\end{array}$ & $\begin{array}{l}\text { Distance } \\
\text { (in) }\end{array}$ & $\begin{array}{l}\text { Media volume/ } \\
\text { Vessel size }(\mathrm{mL})\end{array}$ & $\begin{array}{c}\text { IDR } \pm \text { SD } \\
\left(\mathrm{mg} / \mathrm{cm}^{2} / \mathrm{min}\right)\end{array}$ & $\begin{array}{l}\text { CV } \\
\text { (\%) }\end{array}$ & $R^{2}$ & $\begin{array}{l}\text { IDR percentage } \\
\text { difference (\%) }\end{array}$ & $t$-test $^{b}$ \\
\hline 300 & 5 & 1.0 & $50 / 100$ & $0.546 \pm 0.043$ & 7.84 & 0.9979 & Control & Control \\
\hline \multirow[t]{2}{*}{200} & 5 & 1.0 & $50 / 100$ & $0.576 \pm 0.038$ & 6.53 & 0.9990 & 5.3 & Not significant \\
\hline & 5 & 1.0 & $50 / 100$ & $0.559 \pm 0.019$ & 3.31 & 0.9991 & 2.3 & Not significant \\
\hline \multirow[t]{3}{*}{100} & 5 & 1.5 & $50 / 100$ & $0.458 \pm 0.010$ & 2.15 & 0.9982 & 17.5 & significant \\
\hline & 5 & 1.5 & $100 / 100$ & $0.489 \pm 0.014$ & 2.93 & 0.9987 & 25.3 & Significant \\
\hline & 5 & 1.0 & $50 / 200$ & $0.519 \pm 0.020$ & 3.86 & 0.9974 & Control & Control \\
\hline \multirow[t]{2}{*}{300} & 5 & 1.0 & $100 / 200$ & $0.456 \pm 0.013$ & 2.93 & 0.9978 & 12.9 & Significant \\
\hline & 5 & 1.0 & $200 / 200$ & $0.452 \pm 0.007$ & 1.50 & 0.9993 & 13.9 & Significant \\
\hline
\end{tabular}

${ }^{a}$ Rotation speed: $50 \mathrm{rpm} ; n=6$.

${ }^{b} p<0.05$; IDR of sample is significantly different from that of control. 
the changes in volume of the dissolution medium than by the size of the vessels.

\section{Drug Loading}

The effect of drug loading on IDR of chloramphenicol was also studied using mRDS. As shown in Table 2, the 5-mg drug loading was used as a control for comparing the differences in IDR values. The percentage difference in IDR is less than $5 \%$, and no statistically significant difference was observed for 3-mg and 10-mg as compared with 5-mg drug loading. This indicates that when mRDS was used, the IDR of chloramphenicol was not affected by drug loading.

\section{Rotation Speed}

The effect of rotation speed on IDR of chloramphenicol was investigated using wRDS, mRDS, and mSDS systems. IDR values are directly proportional to the square root of the rotation speed as predicted by $J=0.62^{*} D^{2 / 3} \omega^{1 / 2} v^{-1 / 6} C_{s}$ for the rotating systems (18) and $J=0.77^{*} D^{2 / 3} \omega^{1 / 2} v^{-1 / 6} \gamma^{1 / 2} C_{s}$ for the stationary system (19), where $D$ is the diffusion coefficient, $\omega$ is the angular velocity, $v$ is the kinematic viscosity of the dissolution medium, $\gamma$ is the ratio of the fluid velocity at the axial distance below the paddle to the maximum paddle tip velocity, and $C_{s}$ is the saturated concentration or solubility. As shown in Figure 4 and Table 4, for all three systems, the IDR values increase with the rotation speed. IDR of chloramphenicol is 0.269 (50 rpm), 0.345 (75 rpm), 0.403 (100 rpm), and 0.447 (125 rpm) measured by wRDS; 0.362 (50 rpm), 0.407 (75 rpm), and 0.458 (100 rpm) measured by $\mathrm{mRDS}$; and 0.546 (50 rpm), 0.763 (75 rpm), and 0.944 (100 rpm) measured by mSDS. When the rotation speed increases, the fluid velocity increases, and consequently, the shear at the dissolving surface increases. As a result, the hydrodynamic boundary layer and the diffusion layer on the surface decrease, and the IDR increases (20). The percentage differences in IDR of chloramphenicol measured by WRDS, mRDS, and mSDS with various rotation speeds were compared (Table 4). At the same rotation speed, the IDR appears to be greater with mSDS than with mRDS and wRDS.

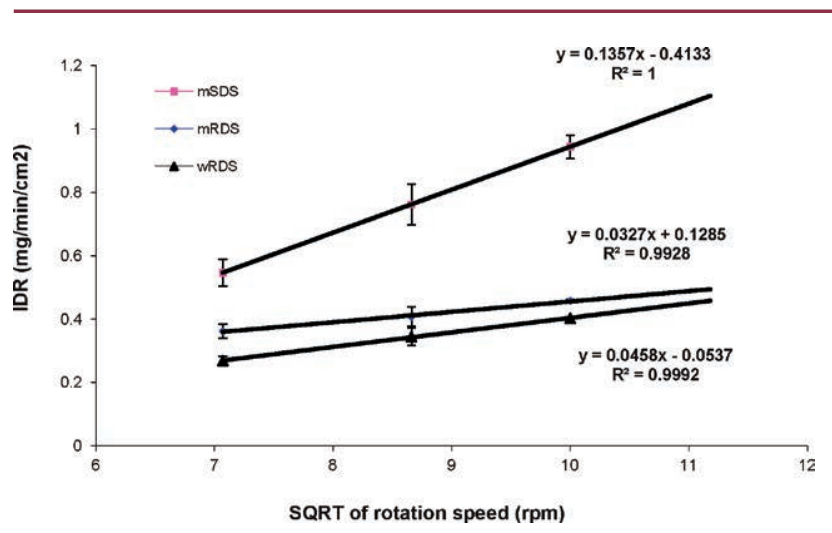

Figure 4. The effect of rotation speed on IDR of chloramphenicol measured by $w R D S, m R D S$, and $m S D S$. Compression force: 300 psi, distance: 1 in, volume/vessel: $50 \mathrm{~mL} / 100 \mathrm{~mL}$, drug loading: $5 \mathrm{mg}, n=6$.

For the stationary system, the disk is in a rotating fluid that sweeps over the stationary dissolution surface agitated by the paddle. At the higher rotation speed, the convection force of the dissolution mechanism outweighs the diffusion force component, which contributes to the considerably increased IDR values for the stationary system $(21,22)$. Since the rotating dissolution surface of the rotating systems is in an unstirred fluid, the influence of the die stirring speed on IDR appears less than that of the paddle agitation. Although the IDR of chloramphenicol measured by mRDS is higher than that of wRDS at the same rotation rate, it is worth noting that it is comparable ( $p>0.05$; no statistically significant difference) to that of wRDS with one level slower rotation speed $(25 \mathrm{rpm})$. This suggests that despite the difference in size of the rotating systems, the mRDS is under similar fluid and hydrodynamic patterns as the standard wRDS.

\section{CONCLUSION}

Two miniaturized apparatus (mRDS and mSDS) were evaluated for measuring IDR values with very small sample sizes (3-10 mg) using chloramphenicol as a model drug. The various experimental parameters, such

Table 4. Percentage Difference in IDR of Chloramphenicol Measured by wRDS, mRDS, and mSDS with Different Rotation Speeds ${ }^{a}$

\begin{tabular}{|c|c|c|c|c|c|c|}
\hline & \multirow{3}{*}{ IDR percentage difference ( $\%$} & & \multicolumn{4}{|c|}{ wRDS } \\
\hline & & & \multicolumn{4}{|c|}{ IDR (Rotation speed, rpm) } \\
\hline & & & $0.269(50)$ & $0.345(75)$ & $0.403(100)$ & $0.447(125)$ \\
\hline \multirow{3}{*}{ mRDS } & \multirow{3}{*}{ IDR (Rotation speed, rpm) } & $0.362(50)$ & 29.4 & $4.8^{b}$ & -10.7 & -20.9 \\
\hline & & $0.407(75)$ & 40.8 & 16.5 & $1.0^{b}$ & -9.2 \\
\hline & & $0.458(100)$ & 52.0 & 28.2 & 12.8 & $2.6^{b}$ \\
\hline \multirow{3}{*}{ mSDS } & \multirow{3}{*}{ IDR (Rotation speed, rpm) } & $0.546(50)$ & 68.0 & 45.2 & 30.2 & 20.1 \\
\hline & & $0.763(75)$ & 95.4 & 75.4 & 61.7 & 52.3 \\
\hline & & $0.944(100)$ & 111.3 & 347.1 & 80.3 & 71.6 \\
\hline
\end{tabular}

a Compression force: 300 psi, distance: 1 in, volume/vessel: $50 \mathrm{~mL} / 100 \mathrm{~mL}$, drug loading: $5 \mathrm{mg} ; n=6$.

${ }^{b} p>0.05$; IDR of samples measured by mRDS is not statistically significant from that measured by wRDS. 
as compression force, disk distance, dissolution volume, and drug loading, had no significant effects on the measured IDR values, demonstrating the robustness of the mRDS system, while dissolution volume and disk distance showed significant effects on IDR of the mSDS system. When comparing the two systems, mSDS may be a better apparatus than mRDS for studying water insoluble compounds with different dissolution mechanisms.

The study shows that the miniaturized disk systems can be useful tools for drug candidate screening when the supplies of bulk drug substances are very limited.

\section{ACKNOWLEDGMENT}

The authors would like to acknowledge Upkar Bhardwaj for his initial assessment work on the stationary disk system (SDS).

\section{REFERENCES}

1. Dressman, J. B.; Amidon, G. L.; Reppas, C.; Shah, V.P. Dissolution Testing as a Prognostic Tool for Oral Drug Absorption: Immediate Release Dosage Forms. Pharm. Res. 1998, 15 (1), 11-22. DOI: 10.1023/A:1011984216775.

2. Amidon, G. L.; Lennernäs, H.; Shah, V. P.; Crison, J. R. A Theoretical Basis for a Biopharmaceutic Drug Classification: The Correlation of In Vitro Drug Product Dissolution and In Vivo Bioavailability. Pharm. Res. 1995, 12 (3), 413-420. DOI: 10.1023/A:1016212804288.

3. Waiver of In Vivo Bioavailability and Bioequivalence Studies for Immediate-Release Solid Oral Dosage Forms Based on a Biopharmaceutics Classification System; Guidance for Industry; U.S. Department of Health and Human Services, Food and Drug Administration, Center for Drug Evaluation and Research (CDER), U.S. Government Printing Office: Washington, DC, 2000.

4. Klein, S. The Mini Paddle Apparatus-a Useful Tool in the Early Development Stage? Experiences with Immediate-Release Dosage Forms. Dissolution Technol. 2006, 13 (4), 6-11.

5. Berge, C. M.; Tsinman, O.; Voloboy, D.; Lipp, D.; Stones, S.; Avdeef, A. Technical Note: Miniaturized Intrinsic Dissolution Rate (Mini-IDR ${ }^{\mathrm{TM}}$ ) Measurement of Griseofulvin and Carbamazepine. Dissolution Technol. 2007, 14 (4), 39-41.

6. Noyes, A. S.; Whitney, W. R. The Rate of Solution of Solid Substances in Their Own Solutions. J. Am. Chem. Soc. 1897, 19 (12), 930-934. DOI: 10.1021/ja02086a003.

7. Viegas, T. X.; Curatella, R. U.; Winkle, L. L. V.; Brinker, G. Measurement of Intrinsic Drug Dissolution Rates Using Two Types of Apparatus. Pharm. Technol. 2001, 25 (6), 44-53.

8. Issa, M. G.; Ferraz, H. G. Intrinsic Dissolution as a Tool for Evaluating Drug Solubility in Accordance with the
Biopharmaceutics Classification System. Dissolution Technol. 2011, 18 (3), 6-13.

9. <1087> Apparent Intrinsic Dissolution- Dissolution Testing Procedures for Rotating Disk and Stationary Disk. In The United States Pharmacopeia and National Formulary USP 35-NF 30; The United States Pharmacopeial Convention, Inc.: Rockville, MD, 2012.

10. 2.9.29. Method of Analysis. Intrinsic Dissolution. In European Pharmacopoeia, 7th ed.; European Directorate for the Quality of Medicines \& Healthcare, Council of Europe: Strasbourg, France, 2011.

11. Appendix XII B. Dissolution ANNEX: Recommendations on Dissolution Testing. In The British Pharmacopoeia; The Stationary Office: London, 2012.

12. Wood, J. H.; Syarto, J. E.; Letterman, H. Improved Holder for Disk Intrinsic Dissolution Rate Studies. J. Pharm. Sci. 1965, 54 (7), 1068. DOI: 10.1002/ jps.2600540730.

13. 4.1.3. Buffer Solutions. In European Pharmacopoeia, 7th ed.; European Directorate for the Quality of Medicines \& Healthcare, Council of Europe: Strasbourg, France, 2011.

14. Banakar, U. V. Pharmaceutical Dissolution Testing; Drugs and the Pharmaceutical Sciences, Vol. 49; Marcel Dekker, Inc.: New York, 1992; pp 19-51.

15. Byrn, S. R.; Pfeiffer, R. R.; Stowell, J. G. Solubility and Dissolution Testing. In Solid-State Chemistry of Drugs, 2nd ed.; SSCI, Inc.: West Lafayette, IN, 1999; pp 91-101.

16. Viegas, T. X.; Curatella, R. U.; Winkle, L. L. V.; Brinker, G. Measurement of Intrinsic Drug Dissolution Rates Using Two Types of Apparatus. Pharm. Technol. 2001, 25 (6), 44-53.

17. Khoury, N.; Mauger, J. W.; Howard, S. Dissolution Rate Studies from a Stationary Disk/Rotating Fluid System. Pharm. Res. 1988, 5 (8), 495-500. DOI: 10.1023/A:1015965223891.

18. Levich, V. G. Physicochemical Hydrodynamics; PrenticeHall: Englewood Cliffs, NJ, 1962; pp 39-72.

19. Colton, C. K.; Smith, K. A. Mass transfer to a rotating fluid. Part II. Transport from the base of an agitated cylindrical tank. AIChE J. 1972, 18 (5), 958-967. DOI: 10.1002/aic.690180512.

20. Mauger, J. W. Physicochemical and Fluid Mechanical Factors Related to Dissolution Testing. Dissolution Technol. 1996, 3 (1), 7-11.

21. Mauger, J.; Ballard, J.; Brockson, R.; De, S.; Gray, V.; Robinson, D. Intrinsic Dissolution Performance Testing of the USP Dissolution Apparatus 2 (Rotating Paddle) Using Modified Salicylic Acid Calibrator Tablets: Proof of Principle. Dissolution Technol. 2003, 10 (3), 6-15.

22. Stevens, L. E. High-Performance Dissolution: the Role of Convective-Diffusion and Controlled Hydrodynamics for Optimal Pharmaceutical Relevance. Am. Pharm. Rev. 2008, 6 (2), 38-45. 